\title{
Therapies and mechanisms of opioid withdrawal
}

\author{
Nicole E Burma ${ }^{1}$, Charlie HT Kwok ${ }^{1}$ \& Tuan Trang ${ }^{*}, 1$ \\ ${ }^{1}$ Comparative Biology \& Experimental Medicine, Faculty of Veterinary Medicine, Physiology \& Pharmacology, Hotchkiss Brain \\ Institute, Cumming School of Medicine, University of Calgary, Calgary, Alberta, T2N 4N1, Canada \\ * Author for correspondence: Tel.: +1 403220 8357; trangt@ucalgary.ca
}

\section{Pain Management}

\author{
"The recent identification of novel cellular substrates of opioid withdrawal that are blocked by \\ clinically approved drugs brings the promise of much needed new therapies."
}

First draft submitted: 4 May 2017; Accepted for publication: 9 June 2017; Published online: 10 November 2017

Keywords: opioid crisis $\bullet$ opioid physical dependence $\bullet$ opioid withdrawal $\bullet$ pharmacological therapies

Opioids are revered as potent analgesics, yet their clinical utility for managing pain is shrouded by concerns over the high abuse potential. With skyrocketing numbers of opioid prescriptions and off-label use, and a striking number of accidental opioid-related deaths and emergency room visits, North America has declared an 'opioid crisis'. In the USA alone, the number of prescribed opioids has quadrupled since 1999, with enough opioid prescriptions dispensed annually to provide a bottle for every household [1]. The opioid crisis, however, is not an isolated North American problem as virtually all developed countries have reported increased opioid consumption and deaths. The alarming rise in opioid-related deaths has stoked growing concerns about the safety of opioids, and increased reluctance on the part of some physicians to prescribe this class of drugs. This has created a conundrum: on one hand, opioids are essential for managing pain, but an over-reliance on opioids can put patients at risk of serious adverse effects, such as opioid analgesic tolerance (diminished pain-relieving effects), hyperalgesia (paradoxical increase in pain sensitivity) and drug dependence (manifesting as drug craving, seeking and/or physical withdrawal).

\section{The problem of opioid withdrawal}

Although adverse effects undermine the long-term clinical utility of opioids for pain management, terminating opioid therapy is also problematic because it can precipitate a debilitating withdrawal syndrome in chronic users. In this respect, opioid withdrawal is a significant challenge in patients where pain has resolved and there is no longer a need for opioid treatment, or those that are on dangerously high doses and a reduction in dose is prudent. Depending on the half-life of the opioid used, withdrawal typically presents between 8 and $48 \mathrm{~h}$ after the last opioid dose, with symptoms including gastrointestinal discomfort, anxiety, insomnia, muscle cramps and hyperhidrosis [2]. Individuals are therefore compelled to continue using opioids to avoid these unpleasant somatic, autonomic and emotional symptoms. Thus opioid withdrawal is a key determinant for continued opioid use and a contributing factor for relapse.

A potential complication of terminating opioid therapy is the re-emergence of pain or exacerbation of a preexisting pain condition. Although pain is a major concern, patients on opioid therapy report that mitigation of withdrawal is a more important consideration for continued opioid use than pain management [3]. However, discerning between pain from a pre-existing condition and pain arising from opioid withdrawal is difficult. Patients may demand higher doses of opioids to manage a perceived worsening of pain, but the underlying reason may stem from withdrawal rather than a progression of a pre-existing pain condition. Withdrawal is also a key motivating factor for continued opioid use in off-label or nonprescription opioid users, and like prescription opioid users, many of these individuals may be better positioned to stop opioid use if more effective treatment options were available for suppressing withdrawal. 


\section{Existing strategies to combat withdrawal in the clinic}

Pharmacological strategies for curbing symptoms of opioid withdrawal are few and far between. Rather than abrupt cessation, the current approach advocates opioid replacement therapy in which the primary goal is to substitute short-acting with longer acting and less euphoric opioids. The driving principle is that opioids with a long half-life produce stable drug plasma levels, circumventing the need for frequent dosing. For this purpose, methadone is commonly prescribed as a replacement opioid requiring a single dose per day to both reduce the incidence of withdrawal and suppress drug cravings. Access to methadone treatment, however, is limited by a shortage in the number of doctors licensed to prescribe methadone. Also, most programs require patients to pick up methadone prescriptions on a day-to-day basis to limit the risk of abuse or overdose, which can introduce significant barriers to individuals living in remote locations or with jobs that require frequent travel.

Buprenorphine/naloxone $\left(\right.$ Suboxone ${ }^{\circledR}$ ) has emerged as an alternative option to methadone for opioid replacement therapy. The combination of buprenorphine, a partial $\mu$-opioid receptor agonist, and naloxone, an opioid receptor antagonist, has conferred several therapeutic advantages over methadone for curbing opioid withdrawal, including an improved safety profile, reduced risk of overdose related death and increased portability [4]. The naloxone component of Suboxone ${ }^{\circledR}$, however, can exacerbate pain symptoms or induce withdrawal in opioid dependent individuals. For chronic pain patients, a further concern is the limited pain-relieving effects of Suboxone ${ }^{\circledR}$, a problem that also impacts individuals on methadone treatment. These patients may remain on opioid substitution therapy for months, years, or in some circumstances indefinitely. Some patients may opt for a gradual and progressive reduction in opioid dose (i.e., opioid tapering). This opioid tapering strategy is intended to reduce or altogether stop opioid use, but can result in withdrawal symptoms that curtail further dose reduction or cause relapse. For a more comprehensive review of therapeutic approaches, including nonopioid adjuvant strategies for managing opioid withdrawal, the reader is referred to other articles on this topic $[2,5]$.

\section{Central mechanisms of opioid withdrawal}

The current opioid crisis has served as a call to battle for scientists and clinicians to develop better therapies for combating opioid withdrawal. This search for novel therapeutic targets is rooted in the principles of opioid pharmacology, which ascribe the actions of opioids to a family of G-protein coupled opioid receptors: $\mu, \delta$ and $\kappa$ that are ever growing in complexity with the discovery of truncated variants and heterogeneity in receptor interactions. While widely distributed in the brain and spinal cord, opioid receptors are particularly concentrated in key sites of the ascending and descending pain pathways, such as in brainstem regions and the superficial dorsal horn. Within the ascending pain pathways (such as in the spinal dorsal horn), opioid receptor activation decreases neuronal excitability through reduced cAMP signalling and voltage-gated calcium channel activity, and through enhanced postsynaptic inward potassium channel currents. Together, this decreases the probability of neurotransmitter release and likelihood of action potential firing in ascending pain circuits. Supraspinally, opioids activate brain regions involved in descending pain control, such as the periaqueductal gray (PAG) and rostral ventromedial medulla, which release monoamines within the spinal cord producing analgesia and suppression of excitatory tone within the nervous system. Furthermore, the locus coeruleus (LC), a central hub from which noradrenergic neurons originate, is densely populated by $\mu$-receptors. Noradrenaline release from the LC is functionally linked to arousal, among other functions, and opioid-receptor activation within the LC counteracts this effect, leading to drowsiness and dampened noradrenergic tone within the central nervous system.

Paradoxically, one of the hallmark features of opioid withdrawal is hyperexcitability of the nervous system. This is thought to be due, in part, to a compensatory change in the expression of proteins directly downstream of opioid receptor activation, such as voltage-gated calcium and potassium channels, and enhanced activation of brain regions tonically suppressed by opioid agonists. For example, tonic inhibition of cAMP signalling and voltagegated calcium channel function with repeated opioid administration causes a compensatory upregulation of these pathways leading to enhanced neuronal excitability, and repeated suppression of LC activity is met by enhanced noradrenergic tone in the absence of an opioid. These changes are unmasked during a state of withdrawal, where the absence of an opioid agonist leads to hyperexcitability of the nervous system and the phenotypic expression of a withdrawal syndrome. It was therefore hypothesized that $\alpha 2$ adrenergic agonists (i.e., clonidine) may be effective at alleviating symptoms of withdrawal, as they directly combat the enhanced noradrenergic tone that results from heightened LC activity. However, the effectiveness of $\alpha 2$ adrenergic agonists is variable between individuals; hence other mechanisms may also contribute to opioid withdrawal. 
Other lines of research also hypothesized that neuronal adaptations occur in response to repeated opioid exposure, and that the mechanisms underlying opioid withdrawal may be similar to those contributing to central sensitization and hyperalgesia in chronic pain states. Indeed, neuronal hyperexcitability within the pain pathway is a key contributor to opioid withdrawal. Several preclinical investigations have indicated that inhibitory transmission in key pain modulatory sites is compromised in animal models of morphine withdrawal. For instance, a reduction in inhibitory potassium channel currents was observed in the PAG [6] and activity of cAMP pathway was upregulated, resulting in increased glutamatergic tone in the rostroventral medulla [7].

At the level of the spinal cord, a key hub for the integration of pain signals, abrupt removal of $\mu$-receptor agonists induces long-lasting synaptic facilitation within the superficial dorsal horn [8]. The interpretation that opioid withdrawal is a result of abnormal neuronal hyperexcitability led to the investigation of several therapeutic strategies, all with the intention to counteract opioid-induced hyperexcitability. Increased levels of extracellular glutamate and NMDAR expression have been observed in several brain regions following naloxone-precipitated withdrawal [9]. While treatment with the NMDAR antagonist ketamine showed promise in alleviating naloxoneprecipitated withdrawal both preclinically and clinically [10,11], exposure to NMDAR antagonists also produced toxic effects similar to those induced by the psychoactive drug phencyclidine (PCP), including sedation, motor depression and hallucination [12]. These collective findings suggest that opioid receptor expressing neurons within the pain pathways are important mechanistic targets for opioid withdrawal.

Apart from the hallmark somatic and autonomic hyperactivity and pain sensitization, there is a large affective component of opioid withdrawal, including mood changes, anxiety and attentional disorders. Hence, brain regions associated with reward and affective behaviors have also been implicated [13]. It has been shown that chronic opioid administration in rodents promoted GABA-mediated excitation [14] and loss of neuronal inhibition via reduction of $\mathrm{KCC}_{2}$ currents in the ventral tegmental area [15]. Furthermore, acute morphine withdrawal in rats increased c-Fos expression in dopaminergic neurons within nucleus accumbens shell in mice [16], and phosphorylation of NMDARs in the accumbens [17]. These studies imply that neuronal hyperexcitability within the limbic regions may alter activity within the dopamine-dependent motivation system, thus producing aversive effects to opioid withdrawal and reinforcing future drug seeking behaviors.

\section{Targeting novel mechanisms: Translating old \& new drugs}

With advances in biochemical processes and increased understanding of opioid pharmacology, more refined ways to eliminate withdrawal were recently proposed. One approach is to manipulate the downstream signalling cascade of opioid receptor activation. Activation of downstream inhibitory G-proteins coupled to opioid receptors recruits the $\beta$-arrestin signalling pathway, which subsequently contributes to the unwanted side-effects of opioid treatment [18]. Using computational screening and structure-based engineering, a new ligand known as PMZ21 was developed, with a powerful ability to activate inhibitory G-proteins with minimal $\beta$-arrestin recruitment [19]. Another approach involved refining the site of opioid action, specifically within the pain pathways. It has been shown that tissue acidosis is a hallmark of tissue injury and pathological pain. This led to the design of a pH-sensitive opioid ligand, known as NFEPP, which selectively activates $\mu$-receptors at the source of pain generation [20]. Both of these ligands show great promise in experimental rodent studies, producing analgesic effects without showing addictive properties. Nonetheless, these recent discoveries will require more rigorous testing in both preclinical and clinical settings.

While the traditional view of opioid withdrawal has been neuron-centric, there is an increasing appreciation that glial-neuron interactions may play a substantial role in opioid withdrawal. Glia were previously thought to function primarily as support cells to neurons, but it is now accepted that they actively react to changes in the environment, releasing key signalling molecules that can modulate neuronal function. For instance, opioid administration causes an increase in microglial and astrocytic reactivity within key sites implicated in opioid withdrawal. Initial studies using a 'broadstroke approach' to block glial function found that co-administration of morphine with AV411, a compound that blocks glial cell reactivity, significantly reduced the physical symptoms of naloxone-precipitated withdrawal [21]. These initial findings sparked a series of related research, further revealing the role of glial cells in opioid withdrawal. Blockade or modulation of proteins expressed on microglia within the PAG [22], nucleus accumbens [23] and spinal cord [24] alleviates the physical symptoms of morphine withdrawal. At the cellular level, blockade of glial function with fluoroacetate significantly reduced long-term potentiation within the spinal dorsal horn following high-frequency stimulation [25], demonstrating a cellular correlate to the influence of glial cell activity over neuronal function. Furthermore, directly blocking specific proteins on microglia, such as the P2X7R 
or Panx1 channel, attenuates high-frequency stimulation-induced long-term potentiation or naloxone-induced facilitation within the spinal dorsal horn [24,25]. The influence of glia on neuronal function is hypothesized to be due to release of key signalling molecules, such as TNF- $\alpha$, which can modulate neuronal surface expression and function of AMPARs and NMDARs [26]. Together, these studies demonstrate the extent of cross-talk between glia and neurons in the central nervous system, and that modulation of glial cell function can significantly regulate the changes in neuronal activity that occurs during opioid withdrawal.

A major challenge in the development of new medications and therapies is the substantial amount of time that lapses between initial bench side discovery and translation to clinical use. As a result, there is increasing investment among researchers to repurpose existing compounds for new clinical applications, as this significantly decreases the time attributed to new drug development, which includes rigorous efficacy and safety testing. In line with preclinical findings that broad-spectrum glial inhibitors are effective at alleviating symptoms of opioid withdrawal, there are ongoing clinical trials testing the effectiveness of minocycline, a tetracycline antibiotic with reported inhibitory effects on glial cell activity, in a human cohort of opioid-dependent patients. Similarly, the clinically available nonselective Panx 1 channel blocker probenecid, currently in use as an anti-gout medication, was found to be effective at alleviating opioid withdrawal in rodents [24]. Furthermore, a recent study by Corder et al. reported that adverse effects of opioid use may be peripherally mediated [27]. The authors showed that co-delivery of a peripherally restricted opioid antagonist, methylnaltrexone bromide, with morphine produced analgesia without analgesic tolerance and opioid-induced hyperalgesia. These clinically approved compounds confer the added benefit of targeting glial pathways or peripheral sites of opioid action, which may circumvent side effects typically associated with the targeting of neuronal pathways (such as NMDARs).

In summary, there is growing awareness and concern on the part of clinicians and patients that long-term opioid use can be associated with severe side-effects. These concerns are magnified by the current opioid crisis, and further complicated by a need for adequate pain management in individuals suffering from chronic pain. A major problem with opioid use is the withdrawal experienced by chronic users. Clinicians have struggled to manage opioid withdrawal given the limited therapeutic options at their disposal, while scientists have grappled to solve its mechanistic puzzle. The recent identification of novel cellular substrates of opioid withdrawal that are blocked by clinically approved drugs brings the promise of much needed new therapies.

\section{Acknowledgements}

The authors would like to thank $\mathrm{H}$ Leduc-Pessah for her helpful comments on the manuscript.

\section{Financial \& competing interests disclosure}

This work was supported by grants from Natural Sciences and Engineering Research Council of Canada, Canadian Institutes of Health Research, Rita Allen Foundation and American Pain Society grant, and Vi Riddell Pain Program grant to T Trang. N Burma is supported by a CIHR Doctoral Scholarship. The authors have no other relevant affiliations or financial involvement with any organization or entity with a financial interest in or financial conflict with the subject matter or materials discussed in the manuscript apart from those disclosed.

No writing assistance was utilized in the production of this manuscript.

\section{References}

1. Paulozzi LJ, Mack KA, Hockenberry JM, Division of Unintentional Injury Prevention, National Center for Injury Prevention and Control, CDC. Vital signs: variation among states in prescribing of opioid pain relievers and benzodiazepines: United States, 2012. MMWR Morb. Mortal. Wkly Rep. 63(26), 563-568 (2014).

2. Clinical Guidelines for Withdrawal Management and Treatment of Drug Dependence in Closed Settings. WHO, Geneva, Switzerland (2009). www.ncbi.nlm.nih.gov/books/NBK310652/

3. Weiss RD, Potter JS, Griffin ML et al. Reasons for opioid use among patients with dependence on prescription opioids: the role of chronic pain. J. Subst. Abuse Treat. 47(2), 140-145 (2014).

4. Alberta College of Pharmacists, College of Pharmacists of British Columbia, Nakatsu N, Sloan M. Principlesfor the Provision of Opioid Dependence Treatment by Manitoba Pharmacists. College of Pharmacists of Manitoba, MB, Canada, 1-49 (2016).

5. Dugosh K, Abraham A, Seymour B, McLoyd K, Chalk M, Festinger D. A systematic review on the use of psychosocial interventions in conjunction with medications for the treatment of opioid addiction. J. Addict. Med. 10(2), 91-101 (2016).

6. Bagley EE, Chieng BCH, Christie MJ, Connor M. Opioid tolerance in periaqueductal gray neurons isolated from mice chronically treated with morphine. Br. J. Pharmacol. 146(1), 68-76 (2005). 
7. Bie B, Peng Y, Zhang Y, Pan ZZ. cAMP-mediated mechanisms for pain sensitization during opioid withdrawal. J. Neurosci. 25(15), 3824-3832 (2005).

8. Drdla R, Gassner M, Gingl E, Sandkühler J. Induction of synaptic long-term potentiation after opioid withdrawal. Science 325(5937), 207-210 (2009).

9. Noda Y, Nabeshima T. Opiate physical dependence and N-methyl-D-aspartate receptors. Eur. J. Pharmacol. 500(1-3), 121-128 (2004).

10. Streel E, Dan B, Antoniali V et al. Effects of anaesthetic agents in interference of naloxone-induced opiate-withdrawal are dose-dependent in opiate-dependent rats. Life Sci. 77(6), 650-655 (2005).

11. Jovaisa T, Laurinenas G, Vosylius S, Sipylaite J, Badaras R, Ivaskevicius J. Effects of ketamine on precipitated opiate withdrawal. Medicina 42(8), 625-634 (2006).

12. Chizh BA, Headley PM. NMDA antagonists and neuropathic pain: multiple drug targets and multiple uses. Curr. Pharm. Des. 11(23), 2977-2994 (2005).

13. Evans CJ, Cahill CM. Neurobiology of opioid dependence in creating addiction vulnerability. F1000Res. 5 , doi:10.12688/f1000research.8369.1 (2016).

14. Vargas-Perez H, Bahi A, Bufalino MR et al. BDNF signaling in the VTA links the drug-dependent state to drug withdrawal aversions. J. Neurosci. 34(23), 7899-7909 (2014).

15. Taylor AMW, Castonguay A, Ghogha A et al. Neuroimmune regulation of GABAergic neurons within the ventral tegmental area during withdrawal from chronic morphine. Neuropsychopharmacol. 41(4), 949-959 (2016).

16. Sharf R, Sarhan M, Dileone RJ. Orexin mediates the expression of precipitated morphine withdrawal and concurrent activation of the nucleus accumbens shell. Biol. Psychiatry 64(3), 175-183 (2008).

17. Anderson EM, Reeves T, Kapernaros K, Neubert JK, Caudle RM. Phosphorylation of the $N$-methyl-D-aspartate receptor is increased in the nucleus accumbens during both acute and extended morphine withdrawal. J. Pharmacol. Exp. Ther. 355(3), 496-505 (2015).

18. Raehal KM, Walker JKL, Bohn LM. Morphine side-effects in beta-arrestin 2 knockout mice. J. Pharmacol. Exp. Ther. 314(3), 1195-1201 (2005).

19. Manglik A, Lin H, Aryal DK et al. Structure-based discovery of opioid analgesics with reduced side effects. Nature 537(7619), 185-190 (2016).

20. Spahn V, Del Vecchio G, Labuz D et al. A nontoxic pain killer designed by modeling of pathological receptor conformations. Science 355(6328), 966-969 (2017).

21. Ledeboer A, Hutchinson MR, Watkins LR, Johnson KW. Ibudilast (AV-411). A new class therapeutic candidate for neuropathic pain and opioid withdrawal syndromes. Expert Opin. Investig. Drugs 16(7), 935-950 (2007).

22. Hutchinson MR, Lewis SS, Coats BD et al. Reduction of opioid withdrawal and potentiation of acute opioid analgesia by systemic AV411 (ibudilast). Brain Behav. Immun. 23(2), 240-250 (2009).

23. Zhang Y, Li H, Li Y et al. Essential role of toll-like receptor 2 in morphine-induced microglia activation in mice. Neurosci. Lett. 489(1), 43-47 (2011).

24. Burma NE, Bonin RP, Leduc-Pessah $\mathrm{H}$ et al. Blocking microglial pannexin-1 channels alleviates morphine withdrawal in rodents. Nat. Med. 23(3), 355-360 (2017).

25. Kronschläger MT, Drdla-Schutting R, Gassner M, Honsek SD, Teuchmann HL, Sandkühler J. Gliogenic LTP spreads widely in nociceptive pathways. Science 354(6316), 1144-1148 (2016).

26. Watkins LR, Hutchinson MR, Milligan ED, Maier SF. 'Listening' and 'talking' to neurons: implications of immune activation for pain control and increasing the efficacy of opioids. Brain Res. Rev. 56(1), 148-169 (2007).

27. Corder G, Tawfik VL, Wang D et al. Loss of $\mu$ opioid receptor signaling in nociceptors, but not microglia, abrogates morphine tolerance without disrupting analgesia. Nat. Med. 23(2), 164-173 (2017). 
